April 9, 2018

MSUHEP-060124

hep-ph/0602211

\title{
Higgs Boson Production and Decay in Little Higgs Models with T-parity
}

\author{
Chuan-Ren Chen, Kazuhiro Tobe and C.-P. Yuan \\ Department of Physics and Astronomy \\ Michigan State University \\ East Lansing, MI 48824, USA
}

\begin{abstract}
We study Higgs boson production and decay in a certain class of Little Higgs models with T-parity in which some T-parity partners of the Standard Model (SM) fermions gain their masses through Yukawa-type couplings. We find that the Higgs boson production cross section of a $120 \mathrm{GeV}$ Higgs boson at the CERN LHC via $g g$ fusion process at one-loop level could be reduced by about $45 \%, 35 \%$ and $20 \%$, as compared to its SM prediction, for a relatively low new particle mass scale $f=$ 600, 700 and $1000 \mathrm{GeV}$, respectively. On the other hand, the weak boson fusion cross section is close to the SM value. Furthermore, the Higgs boson decay branching ratio into di-photon mode can be enhanced by about 35\% in small Higgs mass region in certain case, for the total decay width of Higgs boson in the Little Higgs model is always smaller than that in the SM.
\end{abstract}




\section{Introduction}

In spite of the success of the Standard Model (SM) in describing all the existing experimental data, the Higgs boson, which is responsible for the electroweak symmetry breaking mechanism in the SM, is yet-to-be found at the current Fermilab Tevatron Run II and CERN Large Hadron Collider (LHC) experiments. The elementary Higgs boson in the SM has a difficulty dubbed as "naturalness problem", i.e., its mass parameter can receive huge radiative corrections so that it is difficult to understand why its mass is at the electroweak scale, in contrast to the more fundamental scale, say, Planck scale. Solving the naturalness problem has been one of the driving forces to consider physics models beyond the SM. Supersymmetry [1] is one of the attractive solutions to this problem. All the quadratic divergences in the Higgs mass parameter induced by the SM particles in loops are canceled by those induced by the loops of superpartners of SM particles, and hence the electroweak scale Higgs boson mass parameter is protected from large radiative corrections. Little Higgs mechanism [2] provides another interesting solution. Especially, the large correction induced by top-quark is canceled by that from the heavy fermionic partner of the top-quark, which is originated from the structure of the approximate global symmetry in the top-sector. Similarly, the quadratic divergence in the gauge-boson loops is canceled by that from the extra heavy gauge-bosons which are the partners of SM gauge bosons.

In order to cancel the large quadratic divergence of the Higgs mass parameter, one in general has to introduce new interactions with the Higgs boson. Therefore, studying the consequence of these new Higgs boson interactions would be very important for understanding if such a cancellation is really happening. The new Higgs boson interaction most likely affects $g g \rightarrow h$ production process where the top-Higgs interaction at one-loop level plays a crucial role. It was pointed out in Ref. 3] that the heavy fermionic partner of topquark could modify the Higgs boson production rate via gluon-gluon $(g g)$ fusion process in Little Higgs models. Although many Little Higgs models suffer from strong constraints from precision electroweak measurements [4, recent studies in Refs. [5, 6, 7, 8, 9, 10] have shown that Littlest Higgs models [11] with T-parity have weaker constraints, ${ }^{1}$ and therefore the new particle scale can be significantly smaller than $1 \mathrm{TeV}$.

In this letter, we point out that there are other sets of new Higgs boson interactions in a certain class of Little Higgs models with T-parity. They are needed to generate mass terms for fermionic T-parity partners of the SM fermions, and they can significantly modify the production and decay of Higgs boson at high energy colliders such as the Tevatron and the LHC. The rest of the paper is organized as follows. In Sec. 2, we briefly review the model we study here; a Littlest Higgs model with T-parity. In Sec. 3, we discuss the production rate of Higgs boson via $g g$ fusion process predicted by this model at one-loop level. In Sec. 4, we discuss the other production and decay processes of the

\footnotetext{
${ }^{1}$ The constraints can also be relaxed by adjusting the fermion hypercharges slightly from their original assignments [12].
} 
Higgs boson in this model.

\section{A Littlest Higgs model with T-parity}

The Littlest Higgs model is based on an $S U(5) / S O(5)$ non-linear sigma model [11. A vacuum expectation value $(\mathrm{VEV})$ of an $S U(5) 5 \times 5$ symmetric tensor field $\left(\Sigma_{0}\right)$ breaks the $S U(5)$ to $S O(5)$ at the scale $f$ with

$$
\Sigma_{0}=\left(\begin{array}{lll} 
& & \mathbf{1}_{\mathbf{2} \times \mathbf{2}} \\
& 1 &
\end{array}\right) .
$$

A subgroup $\left[S U(2)_{1} \times U(1)_{1}\right] \times\left[S U(2)_{2} \times U(1)_{2}\right]$ of the $S U(5)$ is gauged, and at the scale $f$ it is broken into the SM electroweak symmetry $S U(2)_{L} \times U(1)_{Y}$. The 14 Goldstone bosons $\Pi^{a}$ associated with this symmetry breaking decompose under $S U(2)_{L} \times U(1)_{Y}$ as $\mathbf{1}_{\mathbf{0}} \oplus \mathbf{3}_{\mathbf{0}} \oplus \mathbf{2}_{\mathbf{1} / \mathbf{2}} \oplus \mathbf{3}_{ \pm \mathbf{1}}$ and they are parametrized by the non-linear sigma model field $\Sigma=\xi^{2} \Sigma_{0}$ as the fluctuations around the VEV in the broken directions, where $\xi=e^{i \Pi^{a} X^{a} / f}$ and $X^{a}$ are the broken generators. The $S U(2)$ doublet in the Nambu-Goldstone multiplet $\Pi^{a}$ is considered to be the Higgs doublet [11].

One way to ensure that the $\rho$-parameter does not deviate from one at tree level (similar to the SM) is to introduce T-parity into the Little Higgs model [5, 6, 7]. Under T-parity, the $\left[S U(2)_{1} \times U(1)_{1}\right]$ gauge fields transform to the $\left[S U(2)_{2} \times U(1)_{2}\right]$ ones, and vice versa. The SM gauge fields are defined as the T-even combination of these gauge fields and the heavy extra gauge bosons are odd under T-parity. Since T-parity is an exact symmetry in this model, the SM gauge bosons do not mix with the T-odd heavy gauge bosons, and consequently, the electroweak precision observables are not modified at tree level. Beyond tree level, small radiative corrections induced by the model to precision data still allow the scale $f$ to be significantly lower than $1 \mathrm{TeV}$ [6, 9]. Moreover, the Little Higgs model with T-parity also provides a possible candidate for dark matter [5, 6, 8, 13].

With T-parity in the Little Higgs models, the SM fermions are T-even and their partners can in general be T-even and/or T-odd. Since we have not seen such kind of T-parity partners, especially T-odd fermions, we need to make them heavy in order to be consistent with experimental measurements. Here, as an interesting example, we consider a Littlest Higgs model with T-parity proposed in Refs. 7, 8, 9] (LH model). (See also the original Littlest Higgs model with T-parity in Ref. [6] and its variation in Ref. [10.)

\subsection{Mass terms for T-odd fermions}

In the LH model [7, 8, 9], two fermion $S U(2)$ doublets $q_{1}$ and $q_{2}$ are introduced in such a way that $q_{A}(A=1,2)$ is transformed as a doublet under $S U(2)_{A}$, and T-parity interchanges these two doublets. The SM $S U(2)$ doublet is taken to be a T-even combination 
of these doublets, and the T-odd combination has to gain a heavy mass. In this section, let us briefly review the mass terms for the T-odd fermions [7, 8, 9].

The fermion $S U(2)$ doublets $q_{1}$ and $q_{2}$ are embedded into incomplete $S U(5)$ multiplets $\Psi_{1}$ and $\Psi_{2}$ as $\Psi_{1}=\left(q_{1}, 0,0_{2}\right)^{\mathrm{T}}$ and $\Psi_{2}=\left(0_{2}, 0, q_{2}\right)^{\mathrm{T}}$, where $0_{2}=(0,0)^{\mathrm{T}}$, and the doublets $q_{1}$ and $q_{2}$ are explicitly written as $q_{A}=-\sigma_{2}\left(u_{L_{A}}, d_{L_{A}}\right)^{\mathrm{T}}=\left(i d_{L A},-i u_{L A}\right)^{\mathrm{T}}$ with $A=1,2$. (The superscript $\mathrm{T}$ denotes taking transpose.) Under a global $S U(5)$ transformation, the multiplets $\Psi_{1}$ and $\Psi_{2}$ transform as $\Psi_{1} \rightarrow V^{*} \Psi_{1}$ and $\Psi_{2} \rightarrow V \Psi_{2}$, where $V$ is an $S U(5)$ rotation matrix. A multiplet $\Psi_{c}$ is also introduced as $\Psi_{c}=\left(q_{c}, \chi_{c}, \tilde{q}_{c}\right)^{\mathrm{T}}$, whose transformation under the $S U(5)$ is non-linear: $\Psi_{c} \rightarrow U \Psi_{c}$. Here $U$ is the unbroken $S O(5)$ rotation and is a non-linear representation of the $S U(5)$. The object $\xi$ and the non-linear sigma model field $\Sigma\left(\equiv \xi^{2} \Sigma_{0}\right)$ transform like $\xi \rightarrow V \xi U^{\dagger}=U \xi \Sigma_{0} V^{T} \Sigma_{0}$ and $\Sigma \rightarrow V \Sigma V^{\mathrm{T}}$, respectively, under $S U(5)$. T-parity is implemented in fermion sector as well as bosonic sector and the transformation laws are defined as follows: $\Psi_{1} \leftrightarrow-\Sigma_{0} \Psi_{2}, \Psi_{c} \rightarrow-\Psi_{c}$, and $\xi \rightarrow \Omega \xi^{\dagger} \Omega$. Thus, $q_{1} \leftrightarrow-q_{2}$ and $\Sigma \rightarrow \tilde{\Sigma} \equiv \Sigma_{0} \Omega \Sigma^{\dagger} \Omega \Sigma_{0}$ under T-parity. Here $\Omega \equiv \operatorname{diag}(1,1,-1,1,1)$. Consequently, the Higgs boson is even under T-parity, and the T-invariant Lagrangian for the mass terms of T-odd fermions can be written as follows:

$$
\mathcal{L}_{\kappa}=-\kappa f\left(\bar{\Psi}_{2} \xi \Psi_{c}+\bar{\Psi}_{1} \Sigma_{0} \Omega \xi^{\dagger} \Omega \Psi_{c}\right)+\text { h.c.. }
$$

Note that $\xi$ contains the Higgs boson $h$, and therefore, this Lagrangian induces new Higgs boson interactions as well as the mass terms for the T-odd fermions:

$$
\mathcal{L}_{\kappa} \simeq-\sqrt{2} \kappa f\left[\bar{d}_{L_{-}} \tilde{d}_{c}+\frac{1+c_{\xi}}{2} \bar{u}_{L_{-}} \tilde{u}_{c}-\frac{s_{\xi}}{\sqrt{2}} \bar{u}_{L_{-}} \chi_{c}-\frac{1-c_{\xi}}{2} \bar{u}_{L_{-}} u_{c}\right]+\text { h.c. } \cdots
$$

Here we only showed the fermion mass terms and a few interaction terms with the neutral Higgs boson. $c_{\xi}\left(\equiv \cos \frac{v+h}{\sqrt{2} f}\right)$ and $s_{\xi}\left(\equiv \sin \frac{v+h}{\sqrt{2} f}\right)$ are originated from the non-linear sigma model field $\xi$, where $h$ and $v$ are neutral Higgs boson field and its VEV, respectively. $u_{L_{-}}$and $u_{L_{+}}$are T-odd and T-even eigenstates, respectively, as defined by $u_{L_{ \pm}}=\left(u_{L_{1}} \mp\right.$ $\left.u_{L_{2}}\right) / \sqrt{2}$. The same definition also applies to the $d$-quark. Moreover, $\tilde{q}_{c}=\left(i \tilde{d}_{c},-i \tilde{u}_{c}\right)^{\mathrm{T}}$, and the second component of the doublet $q_{c}$ is $-i u_{c}$. Note that the T-odd combination of $q_{1}$ and $q_{2}$ together with $\tilde{q}_{c}$ gets a Dirac mass $\sqrt{2} \kappa f$ in a limit of $v \rightarrow 0$, as shown in Eq. (3). We also assume that the Dirac mass terms $\left(-m_{q} \bar{q}_{c}^{\prime} q_{c}-m_{\chi} \bar{\chi}_{c}^{\prime} \chi_{c}\right)$ make the remaining Todd states heavy, and the extra fields $q_{c}^{\prime}$ and $\chi_{c}^{\prime}$ are embedded into a complete $S O(5)$ multiplet, as suggested in Refs. [6, 7, 8, to avoid inducing new quadratic divergence from radiative corrections. The origin of these mass terms may come from new physics above the cutoff scale $4 \pi f$. In our analysis, we simply assume that the masses of these extra particles are larger than the scale $f$. As to be shown later, it turns out that the radiative correction induced by these extra particles to the production cross section of Higgs boson via $g g$ fusion, $\sigma_{g g \rightarrow h}$, is not sensitive to the actual values of these masses, as long as they are much larger than half of the Higgs boson mass. We stress that the Higgs boson interactions in $\mathcal{L}_{\kappa}$ provide $O(1)$ Yukawa-type interactions for $\kappa \sim O(1)$, so that 
these individual interactions could contribute to the quadratic divergences of Higgs boson mass. However, all the quadratic divergences, induced by the individual field introduced in Eq. (2), cancel in the limit of $v \rightarrow 0$, as long as $\Psi_{c}$ forms a complete $S O(5)$ multiplet. Hence, the set of Higgs boson interactions introduced in $\mathcal{L}_{\kappa}$ is consistent with the absence of large quadratic divergences to the Higgs mass parameter. Before we examine the effect of these interactions to the production of Higgs boson via $g g$ fusion, we first review the top Yukawa interactions in the Little Higgs model with T-parity.

\subsection{Top Yukawa interaction}

The large top Yukawa coupling generates a quadratic divergence to the Higgs boson mass. In order to cancel the divergence, one introduces singlet fields $U_{1}$ and $U_{2}$, which are embedded into the doublets: $Q_{1}=\left(q_{1}, U_{1}, 0_{2}\right)^{\mathrm{T}}$ and $Q_{2}=\left(0_{2}, U_{2}, q_{2}\right)^{\mathrm{T}}$, and constructs the following T-parity invariant Lagrangian [7, 8, 9]:

$$
\mathcal{L}_{t}=-\frac{\lambda_{1}}{2 \sqrt{2}} f \epsilon_{i j k} \epsilon_{x y}\left[\left(\bar{Q}_{1}\right)_{i} \Sigma_{j x} \Sigma_{k y}-\left(\bar{Q}_{2} \Sigma_{0}\right)_{i} \tilde{\Sigma}_{j x} \tilde{\Sigma}_{k y}\right] u_{R}-\lambda_{2} f\left(\bar{U}_{1} U_{R_{1}}+\bar{U}_{2} U_{R_{2}}\right)+\text { h.c. }
$$

where $\epsilon_{i j k}$ and $\epsilon_{x y}$ are antisymmetric tensors, and $i, j$ and $k$ run over $1-3$ and $x$ and $y$ over $4-5$. Under T-parity, these fields transform as $Q_{1} \leftrightarrow-\Sigma_{0} Q_{2}, U_{R_{1}} \leftrightarrow-U_{R_{2}}$ and $u_{R} \rightarrow u_{R}$. The above Lagrangian contains the following neutral Higgs boson interactions:

$$
\mathcal{L}_{t} \simeq-\lambda_{1} f\left(\frac{s_{\Sigma}}{\sqrt{2}} \bar{u}_{L_{+}} u_{R}+\frac{1+c_{\Sigma}}{2} \bar{U}_{L_{+}} u_{R}\right)-\lambda_{2} f\left(\bar{U}_{L_{+}} U_{R_{+}}+\bar{U}_{L_{-}} U_{R_{-}}\right)+\text {h.c. },
$$

where $c_{\Sigma}\left(\equiv \cos \frac{\sqrt{2}(v+h)}{f}\right)$ and $s_{\Sigma}\left(\equiv \sin \frac{\sqrt{2}(v+h)}{f}\right)$ are originated from the non-linear sigma model field $\Sigma$. We have defined T-parity eigenstates as $U_{L_{ \pm}}=\frac{U_{1} \mp U_{2}}{\sqrt{2}}$ and $U_{R_{ \pm}}=\frac{U_{R_{1}} \mp U_{R_{2}}}{\sqrt{2}}$. One T-odd Dirac fermion $T^{\prime}\left(T_{L}^{\prime} \equiv U_{L_{-}}, T_{R}^{\prime} \equiv U_{R_{-}}\right)$gets a mass $m_{T^{\prime}}=\lambda_{2} f$ (cf. Eq. (15)), and a T-odd combination of the doublets $q_{1}$ and $q_{2}$ obtains a mass from $\mathcal{L}_{\kappa}$ (cf. Eq. (3) ). Note that $T^{\prime}$ does not have tree level Higgs boson interaction, and thus it does not contribute to the $g g$ fusion process at the one-loop order. The left-handed (or righthanded) top quark is a linear combination of $u_{L_{+}}$and $U_{L_{+}}$(or $u_{R}$ and $\left.U_{R_{+}}\right)$and another independent linear combination is a heavy T-even partner $(T)$ of top quark with the mass

$m_{T} \simeq \sqrt{\lambda_{1}^{2}+\lambda_{2}^{2}} f$ which is responsible for canceling the quadratic divergence to the Higgs mass induced by the top quark.

From Eq. (5), we find that the Higgs boson interactions are approximately given by

$$
-\mathcal{L}=g_{h \bar{t} t} h \bar{t}_{L} t_{R}+g_{h \bar{T} T} h \bar{T}_{L} T_{R}+\text { h.c. },
$$

where

$$
g_{h \bar{t} t} \simeq \frac{m_{t}}{v_{S M}}\left\{1-\frac{3+2 R^{2}+3 R^{4}}{4\left(1+R^{2}\right)^{2}} \frac{v_{S M}^{2}}{f^{2}}+\cdots\right\}, \quad g_{h \bar{T} T} \simeq-\frac{m_{t}}{v_{S M}} \frac{R}{1+R^{2}} \frac{v_{S M}}{f}+\cdots,
$$


with $R=\lambda_{1} / \lambda_{2}$ and $v_{S M} \equiv f \sqrt{1-\cos \frac{\sqrt{2} v}{f}}$. With this definition of $v_{S M}$, gauge boson masses and Fermi constant are expressed, similar to those in the SM, by $m_{Z}=$ $\sqrt{g^{2}+g^{\prime 2}} v_{S M} / 2, m_{W}=g v_{S M} / 2$, and $G_{F}=1 /\left(\sqrt{2} v_{S M}^{2}\right)$ at tree level, respectively. Hence, $v_{S M}$ is equivalent to the Higgs boson VEV in the SM; $v_{S M} \simeq 246 \mathrm{GeV}$. It is important to note that the relation between the top mass and its Yukawa coupling is modified in this model; the top Yukawa coupling is reduced, compared to that in the SM. In addition, the heavy $T$-quark also has Yukawa interaction, but its sign is opposite to that of the top Yukawa coupling. The modification of top-Yukawa coupling and the new Yukawa interaction of $T$-quark will be important for studying the Higgs boson production rate via $g g$ fusion process and the decay branching ratio of Higgs boson into di-photon mode.

\subsection{Other Higgs boson interactions}

Here we summarize other Higgs interactions that are important when we consider Higgs boson productions and decays.

Yukawa couplings of up-type quarks for the first and second generations are given by the similar Lagrangian for the top quark (cf. Eq. (44)), but without introducing extra singlet fields like $U_{i}$ and $U_{R_{i}}(i=1-2)$ in Eq. (4). For down-type quark Yukawa couplings, one of the possible effective Lagrangians [16] is given by

$$
\mathcal{L}_{\text {down }}=\frac{i \lambda_{d}}{2 \sqrt{2}} f \epsilon_{i j} \epsilon_{x y z}\left[\left(\bar{\Psi}_{2}^{\prime}\right)_{x} \Sigma_{i y} \Sigma_{j z} X-\left(\bar{\Psi}_{1}^{\prime} \Sigma_{0}\right)_{x} \tilde{\Sigma}_{i y} \tilde{\Sigma}_{j z} \tilde{X}\right] d_{R}
$$

where $\Psi_{1}^{\prime}=\left(-\sigma_{2} q_{1}, 0,0_{2}\right)^{\mathrm{T}}$ and $\Psi_{2}^{\prime}=\left(0_{2}, 0,-\sigma_{2} q_{2}\right)^{\mathrm{T}}$. Here $X$ transforms into $\tilde{X}$ under T-parity, and it is a singlet under $S U(2)_{i}(i=1-2)$ and its $U(1)_{i}(i=1-2)$ charges are $\left(Y_{1}, Y_{2}\right)=(1 / 10,-1 / 10)$. In this paper, we consider two possible choices $^{2}$ for $X$ : $X=\left(\Sigma_{33}\right)^{-1 / 4}$ (denoted as Case A) and $X=\left(\Sigma_{33}^{\dagger}\right)^{1 / 4}$ (denoted as Case B), where $\Sigma_{33}$ is the $(3,3)$ component of the non-linear sigma model field $\Sigma$.

An important point we note is that all the Yukawa couplings $g_{h \bar{F} F}$ (where $F$ denotes a SM fermion) are modified from those in the $\mathrm{SM}\left(g_{h \bar{F} F}^{\mathrm{SM}}\right)$. Their ratios are approximately given as follows:

$$
\frac{g_{h \bar{u} u}}{g_{h \bar{u} u}^{S M}}=1-\frac{3}{4} \frac{v_{S M}^{2}}{f^{2}}-\frac{5}{32} \frac{v_{S M}^{4}}{f^{4}}+\cdots \simeq\left\{\begin{array}{l}
0.90 \text { for } f=700 \mathrm{GeV}, \\
0.95 \text { for } f=1 \mathrm{TeV},
\end{array}\right.
$$

for $u=$ up and charm quarks (see Eq. (7) for the top Yukawa coupling). For down-type quarks,

$$
\frac{g_{h \bar{d} d}}{g_{h \bar{d} d}^{\mathrm{SM}}}=1-\frac{1}{4} \frac{v_{S M}^{2}}{f^{2}}+\frac{7}{32} \frac{v_{S M}^{4}}{f^{4}}+\cdots \simeq\left\{\begin{array}{l}
0.97 \text { for } f=700 \mathrm{GeV}, \\
0.99 \text { for } f=1 \mathrm{TeV},
\end{array}\right. \text { for Case A, }
$$

${ }^{2}$ We thank Jay Hubisz for pointing out these possibilities to us. The corresponding Lagrangian proposed in Ref. [8], cf. Eq. (2.35), is not invariant under $U(1)_{i}(i=1,2)$. 


$$
=1-\frac{5}{4} \frac{v_{S M}^{2}}{f^{2}}-\frac{17}{32} \frac{v_{S M}^{4}}{f^{4}}+\cdots \simeq\left\{\begin{array}{l}
0.84 \text { for } f=700 \mathrm{GeV}, \\
0.92 \text { for } f=1 \mathrm{TeV},
\end{array}\right. \text { for Case B. }
$$

Note that the down-type quark Yukawa couplings could be significantly suppressed in Case B. We consider the same Yukawa structures in lepton sector, as in quark sector. Thus, the charged lepton Yukawa couplings are also suppressed in the same way as the down-type Yukawa couplings. Similarly, Higgs boson interactions with SM gauge bosons $g_{h V V}$ (where $V=Z, W$ ) are also slightly suppressed:

$$
\frac{g_{h V V}}{g_{h V V}^{\mathrm{SM}}}=1-\frac{1}{4} \frac{v_{S M}^{2}}{f^{2}}-\frac{1}{32} \frac{v_{S M}^{4}}{f^{4}} \cdots \simeq\left\{\begin{array}{l}
0.97 \text { for } f=700 \mathrm{GeV} \\
0.98 \text { for } f=1 \mathrm{TeV}
\end{array}\right.
$$

In addition to the Higgs boson interactions with the SM particles, there are Higgs couplings with heavy extra-gauge bosons and triplet Higgs bosons, as shown in Ref. [3]. All the above mentioned Higgs boson interactions are important for Higgs boson productions and decays as we will discuss later.

\section{$3 \quad$ Higgs boson production via $g g$ fusion process}

\subsection{Contributions from T-odd fermions}

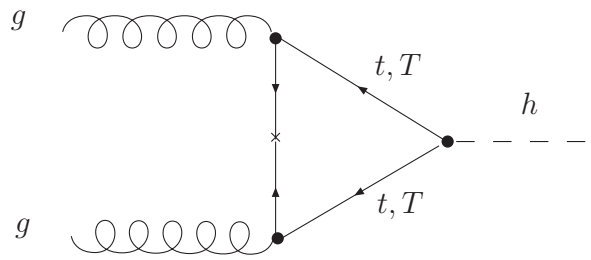

(a)

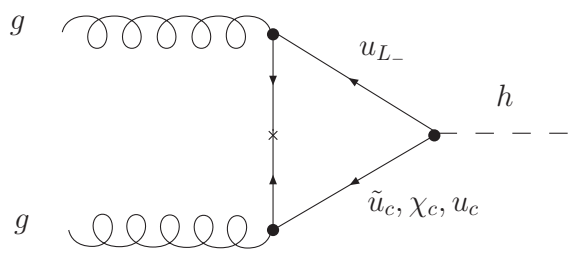

(b)

Figure 1: Contributions to Higgs boson production via $g g$ fusion process $g g \rightarrow h$, induced by (a) top-quark and T-even partner $T$, and (b) T-odd fermions.

Due to the new Higgs boson interaction in $\mathcal{L}_{\kappa}$ of Eq. (3), the T-odd fermions can contribute to the Higgs boson production via $g g$ fusion process at one-loop level, as shown in Fig. 1 (b). From the structure of the mass matrix and Higgs boson interactions for the T-odd fermions, we find that $h \bar{u}_{L_{-}} \tilde{u}_{c}$ interaction provides the dominant T-odd fermion contribution to $\sigma_{g g \rightarrow h}$ and the result is not sensitive to the masses $m_{q}$ and $m_{\chi}$, as long as the T-odd fermion masses are much larger than half of the Higgs boson mass. The ratio of the amplitude induced by the T-odd fermions to the one by the SM top-quark, which is the dominant contribution in the SM, is approximately expressed as

$$
\frac{A_{g g \rightarrow h}(\text { T-odd fermion })}{A_{g g \rightarrow h}(\text { top in SM })} \simeq-\frac{1}{4} \frac{v_{S M}^{2}}{f^{2}}+\cdots \simeq\left\{\begin{array}{l}
-3 \% \text { for } f=700 \mathrm{GeV} \\
-1.5 \% \text { for } f=1 \mathrm{TeV}
\end{array}\right.
$$


Here, we have assumed that the fermions in the loop are much heavier than half of the Higgs boson, so that the one-loop vertex diagram in Fig. 1 can be approximated as a three-point vertex after shrinking the heavy internal lines into a point [17]. The negative sign of the ratio is originated from the positive sign of $h \bar{u}_{L_{-}} \tilde{u}_{c}$ interaction term in Eq. (3) after fixing the correct negative sign for the $\bar{u}_{L_{-}} \tilde{u}_{c^{-}}$mass term. Note that as shown in Eq. (13) the leading order contribution, in terms of $v_{S M} / f$, does not explicitly depend on the parameter $\kappa$. Namely, $\kappa$ term generates a "non-decoupling" contribution to $\sigma_{g g \rightarrow h}$ which does not vanish as $\kappa f \rightarrow \infty$ with a fixed $f$ value. Since the interaction shown in Eq. (2) is needed for each fermion generation to generate mass terms for all the Todd partners, the parameter $\kappa$ has a generation index in general [14. Since the result in Eq. (13) does not depend on $\kappa$, the sum over all three generations of this type of corrections to the $g g \rightarrow h$ amplitude will be three times of the result shown in Eq. (13). (As shown in Eq. (3), there are no equivalent Higgs couplings to down-type quarks in $\mathcal{L}_{\kappa}$.) Hence, the correction $\delta \sigma_{g g \rightarrow h}$ to the production cross section of $g g \rightarrow h$ induced by the T-odd fermions is approximately given by

$$
\frac{\delta \sigma_{g g \rightarrow h}(\text { T-odd fermions })}{\sigma_{g g \rightarrow h}(\text { top in SM })} \simeq-\frac{3}{2} \frac{v_{S M}^{2}}{f^{2}}+\cdots \simeq\left\{\begin{array}{l}
-19 \% \text { for } f=700 \mathrm{GeV}, \\
-9 \% \text { for } f=1 \mathrm{TeV},
\end{array}\right.
$$

for three generation case. Therefore, we find that the effect of the T-odd fermion mass terms on the Higgs boson production rate via $g g$ fusion could be significant, especially when $f$ is below $1 \mathrm{TeV}$.

\subsection{Contributions from top and heavy T-even top partner}

In the SM, the most important contribution to the Higgs boson production via $g g$ fusion process comes from top-quark loop, as shown in Fig. 11 (a). As we have discussed, the top-Yukawa coupling is modified in the LH model, and hence the contribution to the $g g$ fusion process is also modified. Furthermore, there is also new contribution induced at one-loop level by the partner of top-quark, the T-even heavy quark $T$. The ratios of the amplitudes to the top contribution in the SM are given as follows:

$$
\begin{aligned}
& \frac{A_{g g \rightarrow h}(\text { top in LH })}{A_{g g \rightarrow h}(\text { top in SM })} \simeq 1-\frac{3+2 R^{2}+3 R^{4}}{4\left(1+R^{2}\right)^{2}} \frac{v_{S M}^{2}}{f^{2}}+\cdots \\
& \frac{A_{g g \rightarrow h}(T \text { in LH })}{A_{g g \rightarrow h}(\text { top in SM })} \simeq-\frac{m_{t}^{2}}{m_{T}^{2}}+\cdots=-\frac{R^{2}}{\left(1+R^{2}\right)^{2}} \frac{v_{S M}^{2}}{f^{2}}+\cdots,
\end{aligned}
$$

where we have assumed $m_{t}, m_{T} \gg m_{h} / 2$. Therefore, the cross section of the Higgs boson production via $g g$ fusion in the LH model is modified by the T-even top sector (including both top and $T$ contributions). As compared to that in the SM,

$$
\frac{\delta \sigma_{g g \rightarrow h}(\text { T-even top sector })}{\sigma_{g g \rightarrow h}(\text { top in } \mathrm{SM})} \simeq-\frac{3}{2} \frac{v_{S M}^{2}}{f^{2}}+\cdots \simeq\left\{\begin{array}{l}
-19 \% \text { for } f=700 \mathrm{GeV} \\
-9 \% \text { for } f=1 \mathrm{TeV}
\end{array}\right.
$$


We note that although top and $T$ contributions separately depend on $R$, the sum of them does not. This suggests that even if $\lambda_{2}$ is large, and therefore $T$-quark is heavy, the $T$ contribution does not decouple as long as the scale $f$ is about $1 \mathrm{TeV}$. In case of the Littlest Higgs model without T-parity, the authors in Ref. [3] had reached a similar conclusion on the contribution from top and heavy T-even top partner which is rather common in any Littlest Higgs models. ${ }^{3}$ Since the T-odd fermion contribution discussed in the previous section is as large as the one induced by the T-even top-quark sector, the correction to $\sigma_{g g \rightarrow h}$ in the LH model is largely enhanced by the T-odd fermion contributions as compared to that in the Littlest Higgs model without T-parity.

When we sum over all the contributions discussed above, the deviation $\left(\delta \sigma_{g g \rightarrow h} \equiv\right.$ $\left.\sigma_{g g \rightarrow h}^{\mathrm{LH}}-\sigma_{g g \rightarrow h}^{\mathrm{SM}}\right)$ of the Higgs boson production cross section via $g g$ fusion process in the LH model $\left(\sigma_{g g \rightarrow h}^{\mathrm{LH}}\right)$ from the SM prediction $\left(\sigma_{g g \rightarrow h}^{\mathrm{SM}}\right)$ is approximately given by

$$
\frac{\delta \sigma_{g g \rightarrow h}}{\sigma_{g g \rightarrow h}^{S M}} \simeq-3 \frac{v_{S M}^{2}}{f^{2}}+\cdots \simeq\left\{\begin{array}{l}
-37 \% \text { for } f=700 \mathrm{GeV} \\
-18 \% \text { for } f=1 \mathrm{TeV},
\end{array}\right.
$$

where we have assumed that the Higgs mass is smaller than the fermion masses in the loop. It is clear that the extra contributions in the LH model significantly suppress the Higgs boson production cross section via $g g$ fusion process. In Fig. 2, we show a numerical result of the deviation $\left(\delta \sigma_{g g \rightarrow h}\right)$ of the Higgs boson production cross section via $g g \rightarrow h$ in the LH model from the SM prediction, normalized by the SM prediction $\left(\delta \sigma_{g g \rightarrow h} / \sigma_{g g \rightarrow h}^{\mathrm{SM}}\right)$. Here, we assumed that $\kappa=3, m_{q}=m_{\chi}=5 f$ and $R=1$, but we have checked that our result does not strongly depend on these parameters as long as $m_{q}$ and $m_{\chi}$ are much larger than $m_{h} / 2$. For our numerical analysis, we adapted a publically available code, HDECAY [15], for the SM calculation, and modified the code with a complete one-loop calculation in accordance with the effective Lagrangian described in the previous section for the LH model calculation. Dashed lines show the corrections induced by the T-even top sector only (assuming there are no other corrections), and solid lines include the contributions from T-odd fermions in addition to T-even top sector. One sees that the approximate results in Eqs. (14), (17) and (18) well describe the numerical result in Fig. 2 when Higgs mass is small. Fig. [2] show that, if the scale $f$ is smaller than about $1 \mathrm{TeV}$, the production cross section via $g g$ fusion is largely suppressed in all range of Higgs mass, but especially in small Higgs mass region. For example, the deviation from the SM prediction can be more than $40 \%(30 \%)$ for $m_{h}<300 \mathrm{GeV}$ and $f<600(700) \mathrm{GeV}$ if we take into account all the corrections discussed above. This large suppression will be important especially when the Higgs mass is relatively small because the $g g$ fusion process is one of the main discovery modes for detecting a light SM Higgs boson.

\footnotetext{
${ }^{3}$ Since in the Little Higgs model without T-parity, the $\rho$ parameter at tree level is not one 4, the model has stronger constraint on the scale $f$. Therefore, one expects that the effect on the Higgs boson production via $g g$ fusion in the model without T-parity will be much smaller than what is expected in the Littlest Higgs model with T-parity.
} 


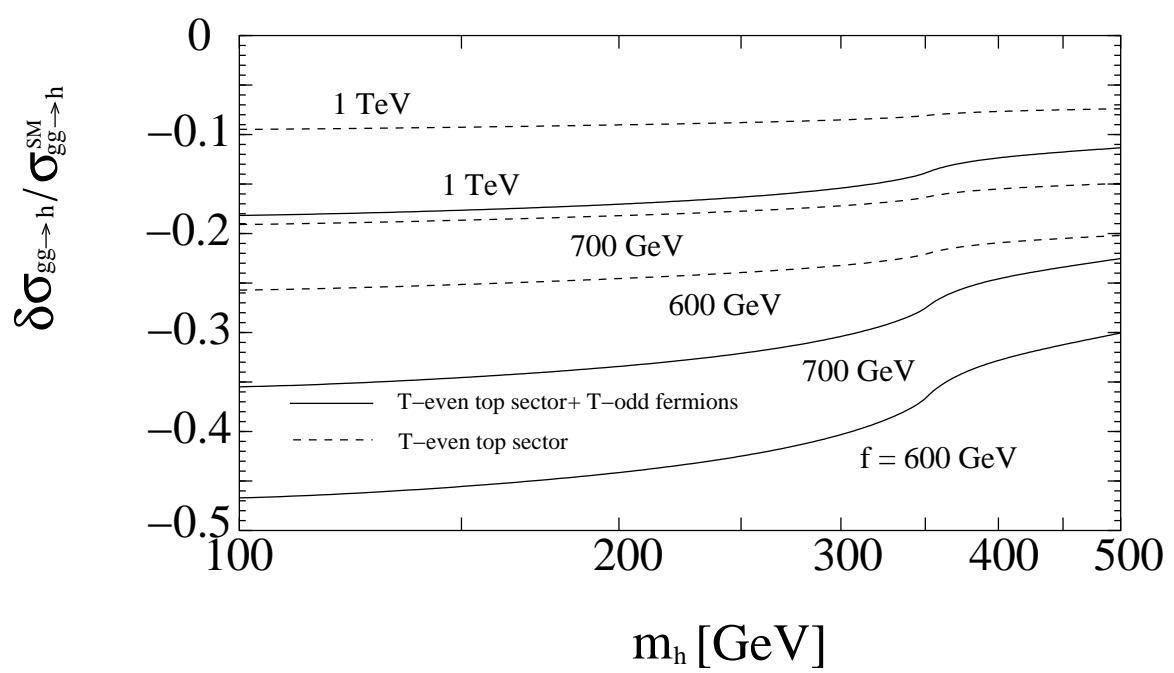

Figure 2: Deviations $\left(\delta \sigma_{g g \rightarrow h}=\sigma_{g g \rightarrow h}^{\mathrm{LH}}-\sigma_{g q \rightarrow h}^{\mathrm{SM}}\right)$ of the Higgs boson production cross section via $g g$ fusion process in the LH model $\left(\sigma_{g g \rightarrow h}^{\mathrm{LH}}\right)$ from that in the SM $\left(\sigma_{g g \rightarrow h}^{\mathrm{SM}}\right)$, normalized by $\sigma_{g g \rightarrow h}^{\mathrm{SM}}$, as a function of Higgs mass $m_{h}$. We have taken $\kappa=3, m_{q}=m_{\chi}=5 f$ and $R=1$, though our result is not sensitive to their specific values as long as $m_{q}, m_{\chi} \gg m_{h} / 2$. Dashed lines show the effect induced by the T-even top sector only. Solid lines include the contributions from T-odd fermions in addition to T-even top sector. In each case, the results for $f=600 \mathrm{GeV}, 700 \mathrm{GeV}$ and $1 \mathrm{TeV}$ are shown. Here, the complete one-loop calculation was used in our numerical analysis.

We note that as suggested by the naive dimensional analysis [18] in low energy effective theory, one may write down the operator, $\sum_{i=1,2} \frac{g_{s}^{2} f^{2}}{\Lambda^{2}} \Sigma_{3 i}^{\dagger} \Sigma_{3 i} G_{\mu \nu}^{A} G^{A, \mu \nu} \sim \frac{g_{s}^{2} h^{\dagger} h}{\Lambda^{2}} G_{\mu \nu}^{A} G^{A, \mu \nu}$, with an $O(1)$ coefficient, where $G_{\mu \nu}^{A}$ is the field strength tensor of the gluon field and $g_{s}$ is the strong coupling. In that case, this operator will induce a counterterm for $h G_{\mu \nu}^{A} G^{A, \mu \nu}$ coupling with its coefficient at the order of $g_{s}^{2} v /\left(16 \pi^{2} f^{2}\right)$, which has the same magnitude as the one-loop contribution calculated above. However, due to the Little Higgs mechanism, we expect the coefficient for the operator $\Sigma_{3 i}^{\dagger} \Sigma_{3 i}$, which generates Higgs boson mass term, to be suppressed by a two-loop suppression factor of $v^{2} / \Lambda^{2} \simeq\left(1 / 16 \pi^{2}\right)^{2}$ as compared to the naive dimensional analysis (with a coefficient $f^{2} \Lambda^{2}$ ). It is likely the same suppression factor $\left(1 / 16 \pi^{2}\right)^{2}$ also applies to the above operator $\Sigma_{3 i}^{\dagger} \Sigma_{3 i} G_{\mu \nu}^{A} G^{A, \mu \nu}$ and yields a much smaller coefficient as compared to the genuine one-loop contributions. Therefore, we expect the one-loop contributions discussed above well represent the dominant contributions to $\sigma_{g g \rightarrow h}$. 


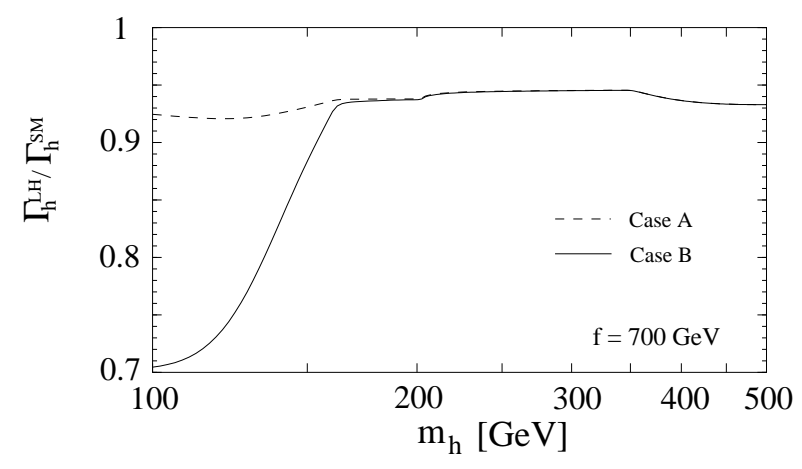

(a)

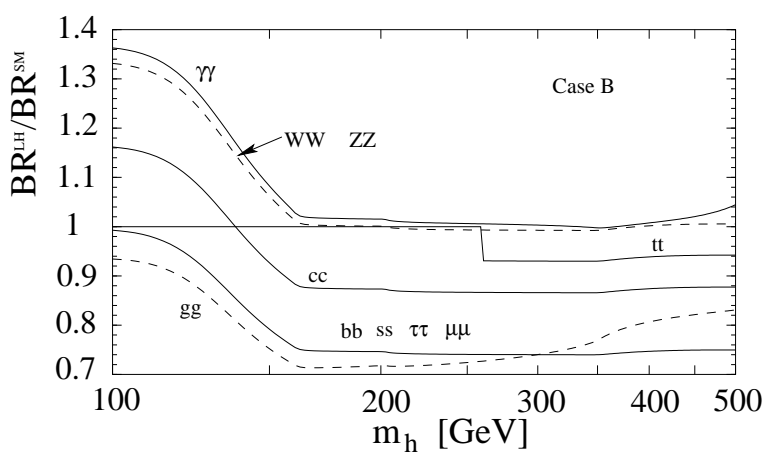

(b)

Figure 3: (a) A ratio of the total Higgs decay width in the LH model $\Gamma_{h}^{\mathrm{LH}}$ to one in the SM $\Gamma_{h}^{\mathrm{SM}}$ for $f=700 \mathrm{GeV}$ in Case $\mathrm{A}$ and B for the down-type quark Yukawa couplings. (b) Ratios of the Higgs decay branching ratios in the LH model $\Gamma_{h}^{\mathrm{LH}}$ to those in the SM $\Gamma_{h}^{\mathrm{SM}}$ for $f=700 \mathrm{GeV}$ in Case B.

\section{Other Higgs boson production channels and decay modes}

In the LH model, the Higgs boson interactions are modified through the interactions of the non-linear sigma model field with other particles, as shown in Eqs. (71), (9), (10), (11) and (12).

Because of the slight suppression of the Higgs couplings with gauge bosons and top quark, the Higgs boson production rates via the gauge boson fusion processes $(V V \rightarrow h$ with $V=W, Z)$, the associated $W h$ and $\bar{t} t h$ processes are also slightly suppressed.

Furthermore, the modification of the Higgs couplings also affects the decay branching ratios of Higgs boson. In Fig. 3 (a), we show the ratios of the total decay width of Higgs boson in the LH model to that in the SM, $\Gamma_{h}^{\mathrm{LH}} / \Gamma_{h}^{\mathrm{SM}}$, for $f=700 \mathrm{GeV}$, in Case A and B of the down-type quark Yukawa interactions, cf. Eq. (8). In Case A, partial decay widths for main decay modes of Higgs boson such as $b \bar{b}, \tau \tau$ and $V V(V=W, Z)$ are almost equally suppressed by about $(0.97)^{2}=0.94$ for $f=700 \mathrm{GeV}$. Therefore, the total decay width of Higgs boson is almost uniformly suppressed in the whole range of Higgs mass. Consequently, the branching ratios of most of the Higgs decay modes are very close to the SM predictions. Thus, in Case A, the only sizable change from the SM prediction is the $g g$ fusion cross section $\sigma_{g g \rightarrow h}$, as discussed in the previous section.

On the other hand, in Case B, both bottom and tau Yukawa couplings are significantly suppressed, and hence the total decay width of Higgs boson is largely reduced in the small Higgs mass region where $h \rightarrow b \bar{b}$ and $\tau \tau$ decay modes dominate, as seen in Fig. 3 (a). For $m_{h}$ larger than $2 m_{W}$, the Higgs boson mostly decays into gauge bosons, $Z Z$ and $W W$, 
and the suppression factor of the total decay width is about $(0.97)^{2}=0.94$ for $f=700$ $\mathrm{GeV}$.

An interesting effect in Case B is that because of the largely reduced total decay width in small Higgs mass region, some of the Higgs boson decay branching ratios are increased even though the corresponding partial decay widths are reduced. We have also calculated the di-photon decay width $\Gamma(h \rightarrow \gamma \gamma)$ at one-loop level. ${ }^{4}$ Similar to the fact that the W-boson contribution dominates over the top-quark contribution to $\Gamma(h \rightarrow \gamma \gamma)$ for small Higgs mass region in the SM, the effect of the extra-fermions in the di-photon decay mode is less important than that in the $g g$ fusion process. Furthermore, the extra-boson contributions tend to cancel the extra-fermion contributions in di-photon decay mode, and therefore the partial decay width of $h \rightarrow \gamma \gamma$ does not change very much, as compared to the SM prediction. As a result, in contrast to Case A, the decay branching ratio of $h \rightarrow \gamma \gamma$ is enhanced by about $35 \%$ for a $100 \mathrm{GeV}$ Higgs boson in Case B, for the total decay width of Higgs boson is reduced by about $30 \%$.

In Fig. 3 (b), we show a numerical result on ratios of Higgs decay branching ratio in the $\mathrm{LH}$ model to that in the SM, BR ${ }^{\mathrm{LH}} / \mathrm{BR}^{\mathrm{SM}}$, for a few main Higgs decay modes in Case B. Here we have again taken $f$ to be $700 \mathrm{GeV}$. We see that especially $\gamma \gamma$ and $V V$ $(V=W, Z)$ decay branching ratios are largely enhanced in the small Higgs mass region.

Since the production cross section via $g g$ fusion is strongly suppressed, the Higgs discovery modes will be changed significantly. In Table $1, R_{\sigma} \times R_{\mathrm{BR}}$ is listed for various production and decay processes in cases for $m_{h}=120 \mathrm{GeV}$ and $200 \mathrm{GeV}$. Here, we define $R_{\sigma(X)}\left(\equiv \sigma_{(X)}^{\mathrm{LH}} / \sigma_{(X)}^{\mathrm{SM}}\right)$ as the ratio of the Higgs boson production cross section in the LH model $\left(\sigma_{(X)}^{\mathrm{LH}}\right)$ to that in the $\mathrm{SM}\left(\sigma_{(X)}^{\mathrm{SM}}\right)$ for each production process $\mathrm{X}$. The subscripts $g g, V V, t \bar{t} h$, and $V h$ represent $g g$ fusion $(g g \rightarrow h)$, weak boson fusion $(V V \rightarrow h$, with $V=W, Z), t \bar{t} h$ and $V h$ associated productions, respectively. $R_{\mathrm{BR}(Y)} \equiv \mathrm{BR}_{(Y)}^{\mathrm{LH}} / \mathrm{BR}_{(Y)}^{\mathrm{SM}}$ for $h \rightarrow Y$ decay modes, where $Y=\gamma \gamma, \tau \tau, b \bar{b}$ and $V V$.

In the SM, the $\gamma \gamma$ decay mode of Higgs boson produced via $g g$ fusion is one of the important discovery channels for a light Higgs boson with mass around $100 \mathrm{GeV}$. However, in the LH model that we study here, this mode would be strongly suppressed. For example, $R_{\sigma(g g)} \times R_{\mathrm{BR}(\gamma \gamma)}=0.68(0.86)$ for $m_{h}=120 \mathrm{GeV}$ and $f=700 \mathrm{GeV}$ in Case A (Case B), as shown in Table 1. Note that since the $h \rightarrow \gamma \gamma$ decay branching ratio is enhanced in Case $\mathrm{B}, R_{\sigma(g g)} \times R_{\mathrm{BR}(\gamma \gamma)}$ is not suppressed as largely as in Case A. The similar conclusion also holds for the $h \rightarrow V V$ mode. On the other hand, $\gamma \gamma$ and $V V$ decay modes of Higgs boson produced via weak boson fusion will be quite different from that via $g g$ fusion since the weak boson fusion process is not largely suppressed. In Case $\mathrm{A}, R_{\sigma(V V)} \times R_{\mathrm{BR}(\gamma \gamma)}$ is very close to the SM prediction. In Case B, however, it could be significantly enhanced because of the enhancement of the $\gamma \gamma$ decay branching ratio. The decay branching ratio to $\tau \tau$ decay mode and the Higgs boson production rate via weak boson fusion do not change in either Case A or Case B. Thus, $R_{\sigma(V V)} \times R_{\mathrm{BR}(\tau \tau)}$ is close to the SM prediction.

\footnotetext{
${ }^{4}$ See Ref. [3] for the study of $h \rightarrow \gamma \gamma$ in the Littlest Higgs model without T-parity.
} 


\begin{tabular}{|r||c|c|c|c|}
\hline$m_{h}=120 \mathrm{GeV}$ & $R_{\mathrm{BR}(\gamma \gamma)}$ & $R_{\mathrm{BR}(\tau \tau)}$ & $R_{\mathrm{BR}(b \bar{b})}$ & $R_{\mathrm{BR}(V V)}$ \\
\hline \hline$R_{\sigma(g g)}($ Case A) & $0.57,0.68,0.84$ & $0.56,0.67,0.83$ & - & $0.55,0.66,0.83$ \\
$($ Case B) & $0.81,0.86,0.93$ & $0.51,0.63,0.81$ & - & $0.78,0.84,0.92$ \\
\hline$R_{\sigma(V V)}($ Case A) & $0.97,0.98,0.99$ & $0.95,0.96,0.98$ & - & $0.94,0.96,0.98$ \\
$($ Case B) & $1.34,1.22,1.09$ & $0.84,0.89,0.95$ & - & $1.30,1.19,1.08$ \\
\hline$R_{\sigma(t \bar{t} h)}($ Case A) & - & $0.87,0.90,0.95$ & $0.87,0.90,0.95$ & - \\
$($ Case B) & - & $0.77,0.83,0.92$ & $0.77,0.83,0.92$ & - \\
\hline$R_{\sigma(V h)}($ Case A) & $0.97,0.98,0.99$ & - & $0.95,0.96,0.98$ & - \\
$($ Case B) & $1.34,1.22,1.09$ & - & $0.84,0.89,0.95$ & - \\
\hline \hline$m_{h}=200 \mathrm{GeV}$ & $R_{\mathrm{BR}(\gamma \gamma)}$ & $R_{\mathrm{BR}(\tau \tau)}$ & $R_{\mathrm{BR}(b \bar{b})}$ & $R_{\mathrm{BR}(V V)}$ \\
\hline \hline$R_{\sigma(g g)}($ Case A) & - & - & - & $0.55,0.67,0.83$ \\
$($ Case B) & - & - & - & $0.56,0.67,0.83$ \\
\hline$R_{\sigma(V V)}($ Case A) & - & - & - & $0.90,0.94,0.97$ \\
$($ Case B $)$ & - & - & $0.90,0.94,0.97$ \\
\hline
\end{tabular}

Table 1: $R_{\sigma} \times R_{\mathrm{BR}}$ for $f=(600,700,1000) \mathrm{GeV}$. Here $R_{\sigma(X)}\left(\equiv \sigma_{(X)}^{\mathrm{LH}} / \sigma_{(X)}^{\mathrm{SM}}\right)$ is defined as a ratio of the Higgs boson production cross section in the little Higgs model $\left(\sigma_{(X)}^{\mathrm{LH}}\right)$ to one in the SM $\left(\sigma_{(X)}^{\mathrm{SM}}\right)$ for each Higgs boson production process $X$. The subscripts $g g, V V$, $t \bar{t} h$, and $V h$ represent $g g$ fusion $(g g \rightarrow h)$, weak boson fusion $(V V \rightarrow h$ where $V=W, Z)$, $t \bar{t} h$ and $V h$ associated productions, respectively. $R_{\mathrm{BR}(Y)} \equiv \mathrm{BR}_{(Y)}^{\mathrm{LH}} / \mathrm{BR}_{(Y)}^{\mathrm{SM}}$ for each Higgs decay mode $h \rightarrow Y$, where $Y=\gamma \gamma, \tau \tau, b \bar{b}$ and $V V$.

When Higgs mass is relatively heavy $\left(m_{h}>160 \mathrm{GeV}\right)$, the decay mode to gauge bosons $(h \rightarrow V V(V=Z, W))$ becomes important. Since in both Case A and Case B the branching ratio for $h \rightarrow V V(V=Z, W)$ is almost the same as the SM prediction, the $V V$ decay mode via gluon fusion production is significantly suppressed, but that via weak boson fusion is not. Therefore, typically the discovery modes of Higgs boson produced via weak boson fusion processes will become more important in the LH model than in the SM. Since these effects could be larger than the experimental uncertainties $(10 \%-20 \%)$ [19] on the measurement of the Higgs boson production cross sections times the branching ratios, they could be observable at the LHC. ${ }^{5}$

We note that when the scale $f$ is around $1 \mathrm{TeV}$, the T-odd heavy gauge boson masses can be of $O(100) \mathrm{GeV}$. For example, for $f=700 \mathrm{GeV}$, the T-odd $U(1)\left(A^{\prime}\right)$ and $S U(2)$ $\left(W^{\prime}\right.$ and $Z^{\prime}$ ) gauge boson masses are $m_{A^{\prime}} \simeq 100 \mathrm{GeV}$ and $m_{W^{\prime}, Z^{\prime}} \simeq 450 \mathrm{GeV}$, respectively. When the Higgs mass is larger than twice of these masses, Higgs boson can decay into the heavy gauge boson pair. We have checked that in that case the decay branching ratios of the heavy gauge boson pair are less than $10^{-2}$ for $f \geq 700 \mathrm{GeV}$. Therefore, the extra

\footnotetext{
${ }^{5}$ We stress that the further improvement of the theoretical calculation will be important to observe these effects.
} 
Higgs decay modes does not significantly change the branching ratios of the SM Higgs boson decay modes.

As discussed in previous sections, the Higgs boson production rate via $g g \rightarrow h$ process in the Littlest Higgs model with T-parity strongly depends on the mechanism to give T-odd particles (and other additional extra-particles) heavy masses. Thus the prediction may be sensitive to new physics above the cutoff scale, and our result shows that the effect could be quite large and become observable at the LHC. Therefore, searching for Higgs boson in various detection modes at the LHC is very important, and the measurement of the relative event rates in multi-channels could reveal the mechanism which provides the cancellation of the quadratic divergence of the Higgs mass parameter and the origin of mass terms for the extra heavy fermions in the LH model.

\section{Acknowledgments}

We thank A. Belyaev, R.S. Chivukula and Jay Hubisz for helpful discussions. This work was supported in part by the U. S. National Science Foundation under award PHY0244919. The Feynman diagrams in Fig. [1] was drawn with JaxoDraw [20].

\section{References}

[1] For reviews, see, for example, H. P. Nilles, Phys. Rept. 110 (1984) 1; H. E. Haber and G. L. Kane, Phys. Rept. 117 (1985) 75; S. P. Martin, arXiv:hep-ph/9709356.

[2] N. Arkani-Hamed, A. G. Cohen and H. Georgi, Phys. Lett. B 513, 232 (2001) For reviews, see, for example, M. Schmaltz and D. Tucker-Smith, arXiv:hep-ph/0502182; M. Perelstein, arXiv:hep-ph/0512128, and references therein.

[3] T. Han, H. E. Logan, B. McElrath and L. T. Wang, Phys. Lett. B 563, 191 (2003) [Erratum-ibid. B 603, 257 (2004)]

[4] C. Csaki, J. Hubisz, G. D. Kribs, P. Meade and J. Terning, Phys. Rev. D 67, 115002 (2003) J. L. Hewett, F. J. Petriello and T. G. Rizzo, JHEP 0310, 062 (2003) C. Csaki, J. Hubisz, G. D. Kribs, P. Meade and J. Terning, Phys. Rev. D 68, 035009 (2003) M. Perelstein, M. E. Peskin and A. Pierce, Phys. Rev. D 69, 075002 (2004) M. C. Chen and S. Dawson, Phys. Rev. D 70, 015003 (2004) W. Kilian and J. Reuter, Phys. Rev. D 70, 015004 (2004) G. Marandella, C. Schappacher and A. Strumia, Phys. Rev. D 72, 035014 (2005)

[5] H. C. Cheng and I. Low, JHEP 0309, 051 (2003)

[6] H. C. Cheng and I. Low, JHEP 0408, 061 (2004) 
[7] I. Low, JHEP 0410, 067 (2004)

[8] J. Hubisz and P. Meade, Phys. Rev. D 71, 035016 (2005)

[9] J. Hubisz, P. Meade, A. Noble and M. Perelstein, arXiv:hep-ph/0506042.

[10] H. C. Cheng, I. Low and L. T. Wang, arXiv:hep-ph/0510225.

[11] N. Arkani-Hamed, A. G. Cohen, E. Katz and A. E. Nelson, JHEP 0207, 034 (2002)

[12] T. Gregoire, D. R. Smith and J. G. Wacker, Phys. Rev. D 69, 115008 (2004) Z. Han and W. Skiba, Phys. Rev. D 72, 035005 (2005)

[13] M. Asano, S. Matsumoto, N. Okada and Y. Okada, arXiv:hep-ph/0602157.

[14] J. Hubisz, S. J. Lee and G. Paz, arXiv:hep-ph/0512169

[15] A. Djouadi, J. Kalinowski and M. Spira, Comput. Phys. Commun. 108, 56 (1998)

[16] J. Hubisz, unpublished work.

[17] J. F. Gunion, H. E. Haber, G. L. Kane and S. Dawson, "The Higgs Hunter's Guide," SCIPP-89/13.

[18] A. Manohar and H. Georgi, Nucl. Phys. B 234, 189 (1984).

[19] D. Zeppenfeld, R. Kinnunen, A. Nikitenko and E. Richter-Was, Phys. Rev. D 62, 013009 (2000) A. Djouadi et al., arXiv:hep-ph/0002258; D. Zeppenfeld, Proc. of the APS/DPF/DPB Summer Study on the Future of Particle Physics (Snowmass 2001) ed. N. Graf, eConf C010630, P123 (2001) arXiv:hep-ph/0203123 ; A. Belyaev and L. Reina, JHEP 0208, 041 (2002) M. Duehrssen, ATL-PHYS-2003-030; M. Duhrssen, S. Heinemeyer, H. Logan, D. Rainwater, G. Weiglein and D. Zeppenfeld, Phys. Rev. D 70, 113009 (2004)

[20] D. Binosi and L. Theussl, Comput. Phys. Commun. 161, 76 (2004) 